\title{
Complications of ultrasound-guided percutaneous microwave ablation of renal cell carcinoma
}

This article was published in the following Dove Press journal:

OncoTargets and Therapy

26 September 2016

Number of times this article has been viewed

\author{
Xuejuan Dong \\ Xin Li \\ Jie Yu \\ Ming-an Yu \\ Xiaoling Yu \\ Ping Liang
}

Department of Interventional Ultrasound, Chinese PLA General Hospital, Beijing, People's Republic of China
Correspondence: Ping Liang

Department of Interventional Ultrasound, Chinese PLA General Hospital,

28 Fuxing Road, Beijing 100853,

People's Republic of China

Tel +861066939530

Email liangping30I@hotmail.com
Purpose: To retrospectively review the complications of ultrasound (US)-guided percutaneous microwave ablation (MWA) of renal cell carcinoma.

Patients and methods: In this study, 101 patients with 105 tumors seen from April 2006 to Feb 2014 were enrolled retrospectively. The patients were treated with US-guided percutaneous MWA and were followed up with contrast-enhanced US and computed tomography or magnetic resonance imaging at 1,3, and 6 months and every 6 months thereafter.

Results: Technical success was achieved in 99 of 105 tumors (94.3\%). The median follow-up time was 25 (range 1.13-93.23) months. Among the 105 tumors, 26 complications in $24.8 \%$ of patients and 23 minor complications (Clavien-Dindo Grades I and II) in 21.9\% of patients were noted, accounting for $88.5 \%$ of all complications. All the minor complications were cured. Three major complications (Clavien-Dindo Grade $\geq$ III) occurred in $2.9 \%$ of the patients, accounting for $11.5 \%$ of all complications: hydrothorax in two patients and bowel injury in one. The two patients who had hydrothorax post-MWA had a history of cirrhosis and were treated with catheter drainage. The bowel injury was treated surgically. In all patients, the changes in serum creatinine and urea nitrogen levels from before to after the procedure were small.

Conclusion: US-guided percutaneous MWA is a beneficial treatment for renal cell carcinoma in selected patients; however, if the renal tumor is close to the bowel, or the patient has serious comorbidities or has undergone abdominal surgery, the procedure must be performed more carefully.

Keywords: microwave ablation, renal cell, complications, carcinoma

\section{Introduction}

Recent advances in surgical techniques have resulted in the replacement of radical nephrectomy with nephron-sparing surgery as the gold standard in the treatment of small renal masses; however, nephron-sparing surgery is associated with increased perioperative complications and patient morbidity. ${ }^{1}$ As a result of the increased incidence of low-stage renal cell cancers (RCCs), thermal ablation technology has emerged as a viable treatment option. Frequently used in situ ablative methods include cryoablation, radiofrequency ablation (RFA), microwave ablation (MWA), and high-intensity focused ultrasound (US). MWA is a minimally invasive treatment that has been used to destroy solid organ malignancies, including RCC. ${ }^{2-4}$ MWA destroys tumors using electromagnetic energy to rotate adjacent polar water molecules within the targeted pathological tissue rapidly, producing heat. ${ }^{5}$ Although RFA is used more widely, MWA has several theoretical advantages over RFA in that it requires a shortened ablation time, produces consistently higher intratumor temperatures, can treat larger ablation volumes, is less dependent on the electrical conductivity of tissue, and has smaller limits on energy delivery because of the exponentially increasing electrical impedance of tumor tissue. ${ }^{6,7}$ 
In recent years, minimally invasive ablation has not only led to shorter hospital stays and enabled the treatment of many patients who were not surgical candidates ${ }^{8}$ but has also reduced complication rates to less than half those of open-surgical approaches (3.1\% vs $7.4 \%$, respectively). ${ }^{9}$ Internationally, RFA and cryoablation are often used to treat RCCs, and their complications are reported more frequently. This paper reports the complications of US-guided percutaneous MWA of RCC.

\section{Materials and methods}

This retrospective study was approved by the review board of the Chinese PLA General Hospital. From April 2006 to February 2014, 111 patients with renal masses underwent US-guided percutaneous MWA in the Department of Interventional Ultrasound, Chinese PLA General Hospital. Written informed consent for the procedure was obtained form all enrolled patients. The patients included in this study met the following criteria: no renal vein embolus or extrarenal metastases; prothrombin time $<25$ seconds, prothrombin activity $>40 \%$, and platelet count $>40 \times 10^{9} / \mathrm{L}$; RCC in solitary kidney; recurrent RCC, which was difficult to operate on; not eligible for surgery or refused to undergo surgery; and presence of an appropriate route for percutaneous puncture using US guidance. Of the 111 patients, 101 patients with 105 tumors were enrolled, while the other ten patients were diagnosed pathologically with benign lesion and thus excluded from the study. All patients were suspected of having RCC and underwent preoperative imaging using contrast-enhanced US and computed tomography (CT) or magnetic resonance imaging (MRI) before ablation. After the results of biopsies were reviewed by two pathologists who had 8 and 10 years of experience, respectively, 92 patients were diagnosed with RCC. Nine patients who could not undergo a biopsy were diagnosed by contrast-enhanced US and CT or MRI.

The information collected from each enrolled patient included demographics, comorbidities, number of tumors, largest tumor diameter, tumor pathology type, tumor growth pattern, tumor adjacent to the intestinal tract or collecting structures, serum creatinine and urea nitrogen levels before and 1 week after MWA, and the date and status at last follow-up. Ablation variables included the time, power, and complications. The growth patterns of the renal nodules were classified as exophytic, parenchymal, or endophytic. Exophytic nodules extend beyond the renal contour without extending into the renal sinus. Parenchymal nodules are restricted within the renal parenchyma. Endophytic nodules violate the renal sinus and are in close proximity to the collecting structures or ureters. If the distance between the tumor margin and bowel was $<5.0 \mathrm{~mm}$, the tumor was categorized as adjacent to the bowel; otherwise, it was categorized as not adjacent to the bowel. The distance was measured by CT, MRI, or US and confirmed by two experienced radiologists using US during MWA, because the distance between the peristaltic bowel and tumor can change with patient position. ${ }^{10}$

\section{US-guided percutaneous MWA}

The KY-2000 microwave unit (Kangyou Medical, Nanjing, People's Republic of China) is capable of producing 0-100 $\mathrm{W}$ power at 2,450 MHz. The needle antenna has a diameter of $1.9 \mathrm{~mm}$ (15 gauge) and a length of $18 \mathrm{~cm}$. The antenna shaft contains two channels through which distilled water at room temperature is passed. After local anesthesia with $1 \%$ lidocaine, a US-guided biopsy was performed before the MWA procedure in all but nine patients. Subsequently, the antenna was inserted into the tumor and was placed at the desired location with US guidance. One antenna was inserted for tumors $<2.0 \mathrm{~cm}$, and two antennas were inserted for those $\geq 2.0 \mathrm{~cm}$. After all insertions were completed, intravenous anesthesia containing a combination of propofol (Diprivan; AstraZeneca plc, London, UK) and ketamine (Shuanghe Pharmaceutical, Beijing, People's Republic of China) was administered. All ablation procedures were performed by two experienced doctors. After MWA, the needle tracks were cauterized to avoid tumor seeding and bleeding. Two to 3 days after ablation, contrast-enhanced imaging (US and CT or MRI) was used to evaluate treatment effectiveness. If the inflammatory congestion caused by MWA was enhanced in scattered, nodular, or irregular areas, the presence of a residual unablated tumor was suspected, and additional MWA was considered if the patient still met the criteria. If the tumor was completely ablated, a well-defined non-enhancing lesion was noted on the contrast-enhanced MRI or CT image and US scans.

\section{Follow-up}

Contrast-enhanced imaging (US and CT imaging or MRI) was performed to evaluate the results of MWA within 1 week after treatment. This was subsequently repeated 1 month after MWA, at 3-month intervals for 1 year, and at 6-month intervals thereafter. Complications were defined as minor (Clavien-Dindo Grades I and II) or major (Clavien-Dindo Grades III, IV, and V). ${ }^{11,12}$ The patients were followed up regularly, and complications were detected using US and CT or MRI and routine blood and urine tests. 


\section{Statistical analyses}

The data were analyzed using SPSS 16.0 (SPSS Inc., Chicago, IL, USA). Patient age, tumor size, and follow-up duration are expressed as means and ranges. Statistical analyses of the effects of MWA on renal function were performed using the $t$-test. Difference with a $P$-value $<0.05$ was considered statistically significant.

\section{Results}

Table 1 summarizes the clinical features of patients and tumors. A total of 119 MWA procedures were performed on 101 patients to treat 105 tumors, and technical success was achieved in 99 of 105 tumors (94.3\%). The median follow-up interval was 25 (range 1.13-93.23) months. The mean age at the time of MWA was 65.4 (range 27-89) years. The mean tumor diameter was 2.9 (range $0.6-8.4$ ) $\mathrm{cm}$, and $17.6 \%$, $55.5 \%$, and $26.9 \%$ of the tumors were located at the upper,

Table I Patient demographics and tumor characteristics of $10 \mathrm{I}$ patients

\begin{tabular}{|c|c|}
\hline & Value \\
\hline \multicolumn{2}{|l|}{ Patient characteristics } \\
\hline Number of patients (\%) & $101(100)$ \\
\hline Male & $75(74.3)$ \\
\hline Female & $26(25.7)$ \\
\hline Median age (years) & $65.4(27-89)^{\mathrm{a}}$ \\
\hline Single lesion & $97(96.0)$ \\
\hline Two lesions & $4(4.0)$ \\
\hline Median follow-up period (months) & $25(1.13-93.23)^{\mathrm{a}}$ \\
\hline \multicolumn{2}{|l|}{ Tumor characteristics } \\
\hline Number of tumors & 105 \\
\hline Tumor diameter $(\mathrm{cm})$ & $2.9(0.6-6)^{\mathrm{a}}$ \\
\hline \multicolumn{2}{|l|}{ Affected side (\%) } \\
\hline Right & $53(50.5)$ \\
\hline Left & $52(49.5)$ \\
\hline \multicolumn{2}{|l|}{ Tumor location (\%) } \\
\hline Upper & $20(19.1)$ \\
\hline Middle & $56(53.3)$ \\
\hline Lower & $29(27.6)$ \\
\hline Adjacent to the bowel & $23(21.9)$ \\
\hline \multicolumn{2}{|l|}{ Growth patterns } \\
\hline Exophytic & $35(33.3)$ \\
\hline Parenchymal & $55(52.4)$ \\
\hline Endophytic & $15(14.3)$ \\
\hline Pathology & 92 \\
\hline Clear cell carcinoma & 82 \\
\hline Papillary carcinoma & 8 \\
\hline Hybrid tumor & 2 \\
\hline Ablation times $(n)$ & 119 \\
\hline $\mathrm{I}$ & 91 \\
\hline 2 & 14 \\
\hline Ablation duration (minutes) & $7.8 \pm 3.6^{\mathrm{b}}(2.5-22.5)^{\mathrm{a}}$ \\
\hline Ablation power (W) & $50.2 \pm 2.9^{\mathrm{b}}(40-60)^{\mathrm{a}}$ \\
\hline
\end{tabular}

Notes: a Data in parentheses are ranges. ${ }^{\circ}$ Data are the mean \pm standard deviation. middle, and lower poles of the kidney, respectively. Of the 101 tumors, 55 (52.6\%) were parenchymal, 35 (33.6\%) were exophytic, and 15 (13.8\%) were endophytic. After pathological examination of tissues, RCC was confirmed in 92 patients, of whom 82 had clear cell carcinoma, eight had papillary carcinoma, and two had a hybrid tumor with scattered areas of oncocytoma and chromophobe carcinoma (Table 1). Nine patients were diagnosed with RCC via imaging studies because three refused biopsies, and six had significant comorbidities contraindicating biopsies. Comorbidities in the 101 patients evaluated included advanced age or poor surgical candidate because of severe cardiovascular disease (46 patients), diabetes mellitus (20), chronic hepatitis and cirrhosis (12), a single kidney (nine), postsurgery kidney carcinoma recurrence (seven), cerebrovascular disease (six), two tumors (six), pneumonectasis, hyperthyroidism, aplastic anemia, and a history of adrenal pheochromocytoma (one each) (Table 2).

Among the 105 renal nodule ablation procedures, 26 complications were noted (Table 3), giving a complication rate of $24.8 \%$. Minor complications (Claviens-Dindo Grades I and II) occurred in $21.9 \%$ of the procedures, and major complications (Clavien-Dindo Grade $\geq$ III) occurred in $2.9 \%$, accounting for $88.5 \%$ and $11.5 \%$ of the overall complications, respectively. The minor complications were as follows: microscopic hematuria (12), mild thermal injury of the psoas muscle (five), perirenal hematoma (two), diarrhea, abdominal distension, edema of the lower limbs, and thermal injury of pelvicalyceal system (one each). All of the minor complications were cured with symptomatic treatment. The major complications were hydrothorax in two patients and bowel injury in one patient. The two patients who had hydrothorax post-MWA had a history of cirrhosis and were treated by catheter drainage.

Table 2 Comorbidities in 101 patients

\begin{tabular}{ll}
\hline Comorbid conditions & $\mathbf{n}$ \\
\hline Severe cardiovascular disease & 46 \\
Chronic hepatitis and cirrhosis & 12 \\
A history of other cancer & 8 \\
Diabetes mellitus & 20 \\
Aplastic anemia & $\mathrm{I}$ \\
Single kidney & 9 \\
Bilateral renal tumor & 6 \\
Kidney carcinoma recurrence postoperation & 7 \\
Severe cerebrovascular disease & 6 \\
Chronic kidney disease & 6 \\
Pneumonectasis & $\mathrm{I}$ \\
Hyperthyroidism & $\mathrm{I}$ \\
A history of adrenal pheochromocytoma & $\mathrm{I}$ \\
\hline
\end{tabular}


Table 3 Complications during and after II 9 renal tumor ablation procedures

\begin{tabular}{lll}
\hline Clavien Grade & $\mathbf{n}$ & Complication \\
\hline I & I2 & Microscopic hematuria \\
& 2 & Perirenal hematoma \\
& 5 & Thermal injury of psoas muscle \\
& I & Thermal injury of pelvicalyceal system \\
II & I & Diarrhea \\
& I & Abdominal distension \\
IIla & I & Edema of lower limbs \\
IIIb & 2 & Hydrothorax \\
IV $/ \mathrm{V}$ & $\mathrm{I}$ & Bowel injury \\
\hline
\end{tabular}

In our series, 23 tumors were adjacent to the bowel, but only one patient, an 84-year-old woman, developed an intestinal perforation post-MWA (Figure 1). She had a history of surgery for ovarian cancer 8 years earlier. Three days after MWA, the patient reported a fever, and occult blood was detected. Gas density in the pelvis and ablated areas was found on CT (Figure 2). Four days later, a gas-fluid level was seen within the urinary bladder, and a CT repeated (Figure 3). The consistency of the patient's urine was similar to that of liquid dung. After a diagnosis of pyelo-ileal internal fistula, she underwent an emergency ileostomy and radical resection of the left kidney (Figure 4). Four months later, the ileostomy was reversed.

In all patients, the serum creatinine and urea nitrogen levels changed slightly post-MWA. The median serum creatinine level was 106.78 (range 39-680.8) $\mu \mathrm{mol} / \mathrm{L}$ pre-MWA and 115.84 (range 40.3-799.9) $\mu \mathrm{mol} / \mathrm{L} 1$ week post-MWA $(P=0.61)$; the normal range is $30-110 \mu \mathrm{mol} / \mathrm{L}$. The mean serum urea nitrogen level was 6.49 (range 3.1-24.87) $\mathrm{mmol} / \mathrm{L}$ pre-MWA and 6.34 (range 2.02-21.93) mmol/L 1 week

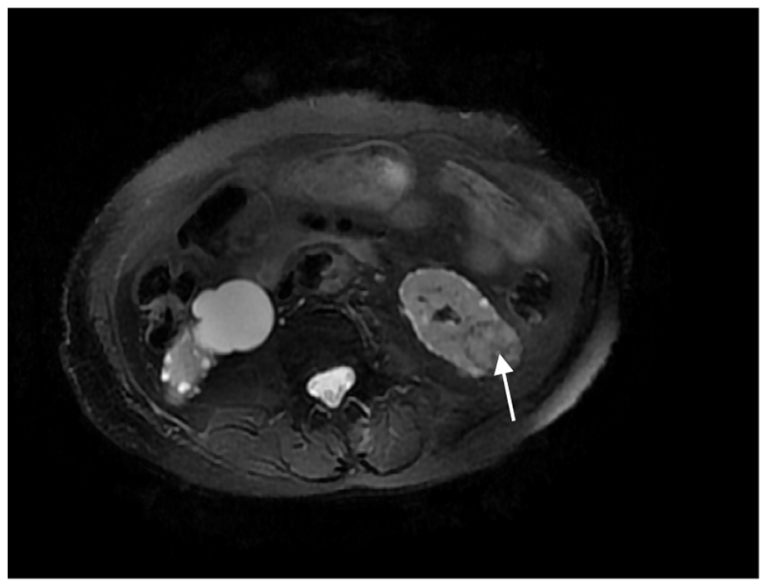

Figure I Magnetic resonance imaging demonstrating left renal nodule adjacent to the bowel before microwave ablation (arrow).

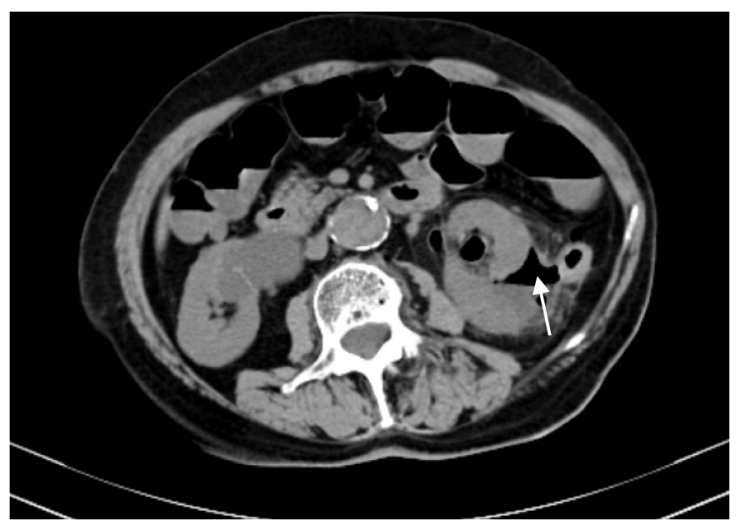

Figure $\mathbf{2}$ The computed tomography scans showing gas density in the ablation areas (arrow).

post-MWA $(P=0.77)$; the normal range is $1.8-7.5 \mathrm{mmol} / \mathrm{L}$. During follow-up, renal function changed slightly in 87 patients with normal renal function pre-MWA and in 14 patients with renal insufficiency pre-MWA.

\section{Discussion}

With advances in imaging techniques, many kidney tumors are discovered during the early stages. This has stimulated the development of minimally invasive treatment options, including RFA and MWA. The objective of the minimally invasive approaches is to preserve renal function and reduce perioperative complications. Although a partial nephrectomy remains the gold standard for RCC, image-guided percutaneous ablation can give similar outcomes with minimal damage.

Compared with RFA, as a form of thermal ablation, MWA is affected less by the perfusion-mediated heat-sink effect, which could help when treating tumors in kidney tissue due to its rich blood supply. ${ }^{13}$ In recent years, MWA has received increasing attention as a promising technique for treating focal malignancies in the liver, kidney, and

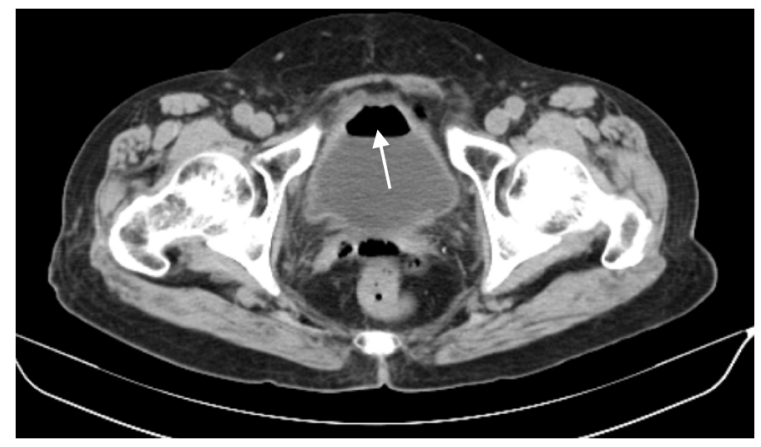

Figure 3 The computed tomography scans showing gas-fluid level within the urinary bladder (arrow). 


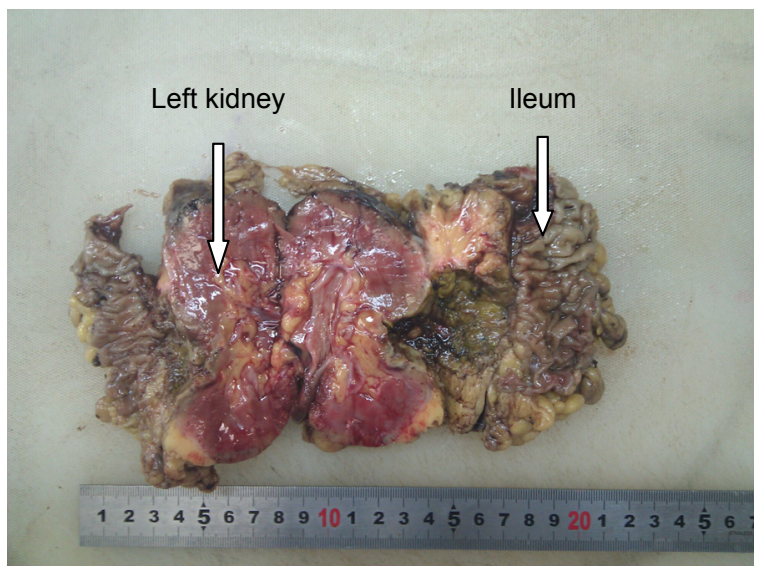

Figure 4 Specimen resected demonstrating partial ileum adhesive to the ablative area of the renal tumor.

other organs. ${ }^{14-16}$ It can achieve higher therapeutic efficacy using various methods. ${ }^{17,18}$

Percutaneous ablation appears to have lower morbidity rates compared with laparoscopic ablation, but the ability to dissect the lesion away from surrounding structures is limited, which can lead to poor outcomes when treating lesions close to the ureter, hilum, or bowel. ${ }^{11}$

Most of the studies show that RFA is efficient, safe, and has a low complication rate. Over the last 10 years, several studies have reported the efficacy and safety of RFA, and long-term studies have confirmed the finding. The overall efficacy of percutaneous ablation for RCC is $90 \%-95 \%$, with a complication rate of $6 \%-7 \% .{ }^{19}$ Complications of RFA include neuralgia, pneumothorax, urine leak, bowel injury, liver burns, massive bleeding, renal insufficiency, transient hematuria, perinephric hematomas, pain or paresthesia, and ureteropelvic junction obstruction. ${ }^{20-22}$ Carrafiello et $\mathrm{al}^{23}$ described "post-RFA syndrome" as consisting of unspecific symptoms such as fever, nausea, vomiting, and/or malaise. More uncommonly, flank wall weakness or laxity has been reported after RFA. ${ }^{24}$

Complications of RFA can generally be divided into those related to imaging-guided electrode placement versus those related to thermal therapy. ${ }^{25}$ The complications of thermal-related injury are more common with kidney RFA than other forms of RFAs, such as hepatic RFA, because the tumor is often adjacent to other vital structures (eg, the bowel and ureter). ${ }^{20}$ Any vital structures near the target are kept at a distance from the ablative zone by maintaining the patient in the prone or lateral decubitus position, as appropriate, after a thorough assessment of the tumor location using preintervention US..$^{20}$ If the distance between the target tissue and tissue requiring protection is too small, invasive methods can be used to dissect the renal mass from the bowel, including hydrodissection or injection of carbon dioxide. ${ }^{26}$

The complications of MWA are similar to those of RFA in the treatment of renal tumors. In this study, follow-up examinations showed that the patient's renal function postMWA was similar to that of pre-MWA, and that any minor complications were temporary and related to the thermal therapy. When the tumor was exophytic, the more common complications were perirenal hematoma and psoas muscle injury; when the tumor was endophytic, hematuria and pelvicalyceal system injury were more likely. Some patients developed nonspecific complications such as diarrhea, abdominal distension, or edema after MWA. The patients with minor complications after MWA recovered within 1 week without the need for any special treatment. The two patients who had hydrothorax post-MWA had a history of cirrhosis and were treated by catheter drainage. In patients who had a renal tumor adjacent to the bowel, hydrodissection was used to dissect the renal mass from the bowel, which was located caudolateral to the renal mass. This method was effective, although one patient developed an intestinal perforation and internal fistula of the ileum and pelvis.

Bowel perforation can occur in RFA if the bowel is contiguous with the ablated tumor, although the insulating effect of perinephric fat generally prevents this complication..$^{26,27}$ When the electrode is sufficiently close enough to the bowel, hyperthermia can injure it, which results in serious complications.

Various techniques have been used to protect injury to nearby structures, including hydrodissection, gas instillation, and balloon instillation. ${ }^{28,29}$ The lever technique can be used to prevent injury to the genitofemoral nerve during RFA of medial kidney tumors by moving the kidney away from the psoas muscle..$^{30}$ The risk of injury to the muscle or nerve is increased in MWA of tumors with exophytic or cortical components in the medial or posterior locations.

In general, hydrodissection is used more frequently, and it might be effective for preventing collateral injury to nearby structures, such as the bowel, psoas muscle, or retroperitoneal nerves. ${ }^{31}$ We performed hydrodissection in 23 patients in whom the renal tumor was close to the bowel before MWA to isolate the bowel and renal lesion. Separation of the gut improved the security of the treatment. Ultimately, only one patient developed a pyelo-ileal internal fistula. Similar complications have been noted previously. ${ }^{21}$ A possible reason for this complication was that although hydrodissection was performed prior to MWA, the patient had previous abdominal 
viscera adhesions such that the bowel was not at a sufficient distance from the ablative field to avoid damage. The vital structures remained near the ablative field, which resulted in severe intestinal injury. We believe that the complication rate could decrease significantly with stringent patient selection based on patient and tumor characteristics. ${ }^{24}$ We should consider not only paying attention to the location of the tumor and nearby organs but also asking for details of the patient's medical history and treatment method, in order to fully assess the risk of treatment, and formulate a reasonable treatment plan.

\section{Limitations}

This study had some limitations. First, its retrospective nature might have influenced the evaluation of clinical outcomes. Second, the number of patients was small, especially that of patients with tumors adjacent to important structures, such as the bowel or ureter. It was difficult to analyze the risk factors for each complication. Third, this was a single-center study, and thus, the clinical experience of these doctors might have directly influenced the results.

\section{Conclusion}

US-guided percutaneous MWA is useful in the treatment of RCC in selected patients, and it can be performed in patients with serious comorbidities. The complication rate is low, and most of the complications are minor and easily treatable. However, if the renal tumor is close to the bowel or the patient has serious comorbidities or a history of abdominal surgery, the procedure must be performed more carefully.

\section{Acknowledgment}

The authors thank Meng-juan Mu for her assistance with writing this manuscript.

\section{Author contributions}

Xuejuan Dong and Ping Liang carried out the literature research. Xuejuan Dong, Xin Li, Ming-an Yu, and Ping Liang conducted the clinical studies. Xuejuan Dong, Xin Li, Jie Yu, and Ming-an Yu performed the statistical analysis. Xuejuan Dong edited the manuscript. All authors contributed toward data analysis, drafting and revising the paper and agree to be accountable for all aspects of the work.

\section{Disclosure}

The authors have declared there are no conflicts of interest with regards to this work.

\section{References}

1. Gill IS, Kavoussi LR, Lane BR, et al. Comparison of 1,800 laparoscopic and open partial nephrectomies for single renal tumors. J Urol. 2007;178(1):41-46.

2. Nahum Goldberg S, Dupuy DE. Image-guided radiofrequency tumor ablation: challenges and opportunities - part I. J Vasc Interv Radiol. 2001;12(9):1021-1032.

3. Dodd GD 3rd, Soulen MC, Kane RA, et al. Minimally invasive treatment of malignant hepatic tumors: at the threshold of a major breakthrough. Radiographics. 2000;20(1):9-27.

4. Gazelle GS, Goldberg SN, Solbiati L, Livraghi T. Tumor ablation with radio-frequency energy. Radiology. 2000;217(3):633-646.

5. Goldberg SN, Gazelle GS, Mueller PR. Thermal ablation therapy for focal malignancy: a unified approach to underlying principles, techniques, and diagnostic imaging guidance. AJR Am J Roentgenol. 2000;174(2):323-331.

6. Brace CL. Radiofrequency and microwave ablation of the liver, lung, kidney, and bone: what are the differences? Curr Probl Diagn Radiol. 2009;38(3):135-143.

7. Simon CJ, Dupuy DE, Mayo-Smith WW. Microwave ablation: principles and applications. Radiographics. 2005;25(Suppl 1): S69-S83.

8. Hui GC, Tuncali K, Tatli S, Morrison PR, Silverman SG. Comparison of percutaneous and surgical approaches to renal tumor ablation: metaanalysis of effectiveness and complication rates. $J$ Vasc Interv Radiol. 2008;19(9):1311-1320.

9. Howenstein MJ, Sato KT. Complications of radiofrequency ablation of hepatic, pulmonary, and renal neoplasms. Semin Intervent Radiol. 2010;27(3):285-295.

10. Yu J, Liang P, Yu XL, et al. US-guided percutaneous microwave ablation of renal cell carcinoma: intermediate-term results. Radiology. 2012;263(3):900-908.

11. Kim SD, Yoon SG, Sung GT. Radiofrequency ablation of renal tumors: four-year follow-up results in 47 patients. Korean J Radiol. 2012;13(5):625-633.

12. Dindo D, Demartines N, Clavien PA. Classification of surgical complications: a new proposal with evaluation in a cohort of 6336 patients and results of a survey. Ann Surg. 2004;240(2):205-213.

13. Wright AS, Sampson LA, Warner TF, Mahvi DM, Lee FT Jr. Radiofrequency versus microwave ablation in a hepatic porcine model. Radiology. 2005;236(1):132-139.

14. Liang P, Wang Y. Microwave ablation of hepatocellular carcinoma. Oncology. 2007;72(Suppl 1):124-131.

15. Wang Y, Liang P, Yu X, Cheng Z, Yu J, Dong J. Ultrasound-guided percutaneous microwave ablation of adrenal metastasis: preliminary results. Int J Hyperthermia. 2009;25(6):455-461.

16. Liang P, Wang Y, Zhang D, Yu X, Gao Y, Ni X. Ultrasound guided percutaneous microwave ablation for small renal cancer: initial experience. $J$ Urol. 2008;180(3):844-848.

17. Lin Y, Liang P, Yu XL, et al. Percutaneous microwave ablation of renal cell carcinoma is safe in patients with a solitary kidney. Urology. 2014;83(2):357-363.

18. Dong B, Liang P, Yu X, et al. Percutaneous sonographically guided microwave coagulation therapy for hepatocellular carcinoma: results in 234 patients. AJR Am J Roentgenol. 2003;180(6):1547-1555.

19. Georgiades $\mathrm{C}$, Rodriguez R. Renal tumor ablation. Tech Vasc Interv Radiol. 2013;16(4):230-238.

20. Park BK, Kim CK. Complications of image-guided radiofrequency ablation of renal cell carcinoma: causes, imaging features and prevention methods. Eur Radiol. 2009;19(9):2180-2190.

21. Boone J, Bex A, Prevoo W. Percutaneous radiofrequency ablation of a small renal mass complicated by appendiceal perforation. Cardiovasc Intervent Radiol. 2012;35(3):695-699.

22. Barreto F, Dall'Oglio MF, Srougi M. Acute renal insufficiency after radiofrequency of renal tumor. Int Braz J Urol. 2007;33(5):679-681; discussion 682 . 
23. Carrafiello G, Lagana D, Ianniello A, et al. Post-radiofrequency ablation syndrome after percutaneous radiofrequency of abdominal tumours: one centre experience and review of published works. Australas Radiol. 2007;51(6):550-554.

24. Bhayani SB, Allaf ME, Su LM, Solomon SB. Neuromuscular complications after percutaneous radiofrequency ablation of renal tumors. Urology. 2005;65(3):592.

25. Rhim H, Dodd GD 3rd, Chintapalli KN, et al. Radiofrequency thermal ablation of abdominal tumors: lessons learned from complications. Radiographics. 2004;24(1):41-52.

26. Ginat DT, Saad W, Davies M, Walman D, Erturk E. Bowel displacement for CT-guided tumor radiofrequency ablation: techniques and anatomic considerations. J Endourol. 2009;23(8):1259-1264.

27. Clark TW, Malkowicz B, Stavropoulos SW, et al. Radiofrequency ablation of small renal cell carcinomas using multitined expandable electrodes: preliminary experience. J Vasc Interv Radiol. 2006;17(3): 513-519.
28. Farrell MA, Charboneau JW, Callstrom MR, Reading CC, Engen DE, Blute ML. Paranephric water instillation: a technique to prevent bowel injury during percutaneous renal radiofrequency ablation. AJR Am J Roentgenol. 2003;181(5):1315-1317.

29. Kam AW, Littrup PJ, Walther MM, Hvizda J, Wood BJ. Thermal protection during percutaneous thermal ablation of renal cell carcinoma. J Vasc Interv Radiol. 2004;15(7):753-758.

30. Boss A, Clasen S, Kuczyk M, et al. Thermal damage of the genitofemoral nerve due to radiofrequency ablation of renal cell carcinoma: a potentially avoidable complication. AJR Am J Roentgenol. 2005;185(6): $1627-1631$.

31. Lee SJ, Choyke LT, Locklin JK, Wood BJ. Use of hydrodissection to prevent nerve and muscular damage during radiofrequency ablation of kidney tumors. J Vasc Interv Radiol. 2006;17(12):1967-1969.
OncoTargets and Therapy

\section{Publish your work in this journal}

OncoTargets and Therapy is an international, peer-reviewed, open access journal focusing on the pathological basis of all cancers, potential targets for therapy and treatment protocols employed to improve the management of cancer patients. The journal also focuses on the impact of management programs and new therapeutic agents and protocols on

\section{Dovepress}

patient perspectives such as quality of life, adherence and satisfaction. The manuscript management system is completely online and includes a very quick and fair peer-review system, which is all easy to use. Visit http://www.dovepress.com/testimonials.php to read real quotes from published authors.

Submit your manuscript here: http://www.dovepress.com/oncotargets-and-therapy-journal 